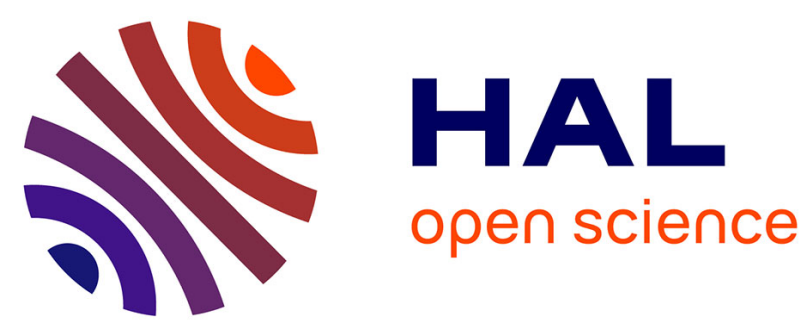

\title{
Capillary self-alignment assisted hybrid robotic Handling for Ultra-Thin Die Stacking.
}

Ville Liimatainen, Mohamed Kharboutly, David Rostoucher, Michaël Gauthier, Quan Zhou

\section{To cite this version:}

Ville Liimatainen, Mohamed Kharboutly, David Rostoucher, Michaël Gauthier, Quan Zhou. Capillary self-alignment assisted hybrid robotic Handling for Ultra-Thin Die Stacking.. IEEE International Conference on Robotics and Automation (ICRA), Jan 2013, Finland. pp.1395 - 1400. hal-00833834

\section{HAL Id: hal-00833834 \\ https://hal.science/hal-00833834}

Submitted on 13 Jun 2013

HAL is a multi-disciplinary open access archive for the deposit and dissemination of scientific research documents, whether they are published or not. The documents may come from teaching and research institutions in France or abroad, or from public or private research centers.
L'archive ouverte pluridisciplinaire HAL, est destinée au dépôt et à la diffusion de documents scientifiques de niveau recherche, publiés ou non, émanant des établissements d'enseignement et de recherche français ou étrangers, des laboratoires publics ou privés. 


\title{
Capillary Self-Alignment Assisted Hybrid Robotic Handling for Ultra-Thin Die Stacking
}

\author{
Ville Liimatainen ${ }^{1}$, Mohamed Kharboutly², David Rostoucher ${ }^{2}$, Michaël Gauthier ${ }^{2}$, Member, IEEE, \\ and Quan Zhou ${ }^{1}$, Member, IEEE.
}

\begin{abstract}
Ultra-thin dies are difficult to package because of their fragility and flexibility. Current ultra-thin die integration technology for 3D microsystems relies on robotic pick-andplace machines and machine vision, which has rather limited throughput for high-accuracy assembly of fragile ultra-thin dies. In this paper, we report a hybrid assembly strategy that consists of robotic pick-and-place using a vacuum microgripper, and droplet self-alignment by capillary force. Ultrathin dies with breakable links are chosen as part of the assembly strategy. Experimental results show that we can align ultra-thin $(10 \mu \mathrm{m})$ dies with sub-micron accuracy without machine vision. A fully automatic sequence of stacking several of these dies is demonstrated. Up to 12 ultra-thin dies have been stacked. These early results show that die-to-die integration of ultra-thin dies with higher throughput than the current industry robot is possible by applying both robotic handling and droplet self-alignment to ultra-thin die assembly.
\end{abstract}

\section{INTRODUCTION}

Ultra-thin dies are thinned semiconductor chips having thickness below e.g. $20 \mu \mathrm{m}$, which is an evolving value as the technology progresses. The primary need for ultra-thin die fabrication and assembly techniques is in $3 \mathrm{D}$ integration, where different active dies are vertically connected in stacks using through-silicon-vias (TSV). Ultra-thin dies offer several benefits for such die stacks. They enable lower package height for thin integrated circuits (IC) applications, enhanced heat flow for high power components, shorter interconnections for faster operation and easier TSV formation due to reduced thickness.

One great challenge in ultra-thin die stacking is to fulfill the requirement of high-accuracy and high-throughput simultaneously, while avoiding damage to the dies. For 3D die stacks, the alignment accuracy of TSVs between the dies is crucial to ensure working electrical connections. According to the ITRS roadmap for 3D die stacks, the required alignment accuracies are approaching the submicron level while the number of aligned layers is increasing beyond two [1]. Currently tools such as flip-chip die bonder are used for stacking thin dies using machine vision based positioning technology. In addition to accuracy, which translates to yield, fast assembly is essential for costeffectiveness. Current state-of-the-art die bonding machines can achieve very high throughput when the positioning accuracy is modest, but when the accuracy requirement increases, the throughput decreases. For example, sub-micron

\footnotetext{
Aalto University, School of Electrical Engineering, Department of Automation and Systems Technology, Otaniementie 17, 02150 Espoo, Finland

2 FEMTO-ST Institute, AS2M dept. UMR CNRS 6174 UFC/ENSMM/UTBM, 24 rue Alain Savary, 25000, Besançon, France
}

accuracy in the assembly of thick dies is achievable on commercial systems, but with very low throughput of only a couple of hundred dies per hour [2]. For ultra-thin dies the throughput is further limited due to fragility and flexibility of the dies.

To tackle the throughput and accuracy dilemma, we use an assembly method for ultra-thin dies based on capillary self-alignment assisted robotic pick-and-place. The method is built upon the recent efforts on combining robotic microhandling and self-assembly into a hybrid microassembly strategy [3-7], where fast-speed robotic pick-and-place is used for coarse positioning, and high accuracy is reached by capillary self-alignment of a droplet, driven by the capillary force between the die and the receptor site. The capillary force also aids in overcoming the adhesion forces between the die and the gripper, which is a common problem in micromanipulation. Despite previous studies has been carried out on hybrid microassembly, the ultra-thin die stacking brings many new challenges to be tackled, including the handling of fragile dies, the potential effect of such dies on self-alignment, and the question if the required accuracy can be achieved for ultra-thin dies.

To avoid damaging the fragile dies during handling, the ultra-thin dies are prepared in a silicon layer with breakable links, similar to [8]. Since this study is the first step in investigation of this method, only dummy dies are used. Vacuum tools and precision robotic positioning system is used for pick-and-place of the dies. To achieve good alignment in die stacking, we investigated several water droplet self-alignment approaches. And finally, we have stacked up to 12 ultra-thin dies to demonstrate the feasibility of the approach.

\section{UltRA-THIN DIES WITH BREAKABLE LINKS}

To fabricate ultra-thin dies, the wafer must be thinned. Conventionally, thinning of wafers is done by grinding the back side of the wafer [9]. This mechanical grinding can be combined with polishing like in the traditional chemicalmechanical-polishing method (CMP), or the surface can be finished after grinding by wet etching or dry plasma etching. The dies are then singulated by sawing on a thermal or UV sensitive dicing tape, or by DRIE etching on a carrier wafer. More recently, the process has been altered so that dicing happens as a result of grinding the wafer backside until precut grooves on the front side are reached. Plasma etching is then normally used for finishing the ground surface and for stress relief of the dies.

The challenge with the thinning and singulation processes is that they damage the dies in several ways [10]. The situation gets worse when the dies become thinner, and the 
problem extends to picking them up intact from a temporary carrier tape or wafer. Indeed, in the case of a die with thickness around $10 \mu \mathrm{m}$, the force required to remove it from adhesive tape may be higher than the mechanical rupture force of the die. To bypass these problems, we fabricate the dies on the device layer of an SOI wafer by etching around the dies, leaving them connected to the background only at one point, using a technique similar to [11]. This connection serves as a breakable link, as presented in Fig. 1.

The dies are fabricated in three main steps (Fig. 2). First, the handle layer (typically $400 \mu \mathrm{m}$ thick) of an SOI wafer is etched through until the silicon dioxide insulator layer by deep reactive-ion etching (DRIE), as shown in Fig. 2 (Step 1). Then the device layer is patterned and etched in the shape of the dies, leaving narrow bridges to connect the dies to the background (Fig. 2, Step 2). Finally, the intermediate silicon dioxide layer is removed using hydrogen-fluoride (HF) vapor and the dies are left hanging on the breakable links (Fig 2, Step 3). The structure and dimensions of the links (Fig.1, highlighted in red) have been designed so that the rupture during cracking is confined and does not damage the die, as explained in the next section.

\section{METHODS AND TOOLS}

The die and breakable link structure must respect three main mechanical constraints. First, it should be strong enough to survive the fabrication process. Second, the link should break when the die is pushed vertically down by a vacuum gripper head. Third, the die should withstand the suction force applied by the vacuum gripper during pick-andplace operation. These constraints, summarized in Fig. 3, were determined by estimation, simulation and measurements. The same constraints were also considered in the design of a suitable vacuum gripper and handling strategy.

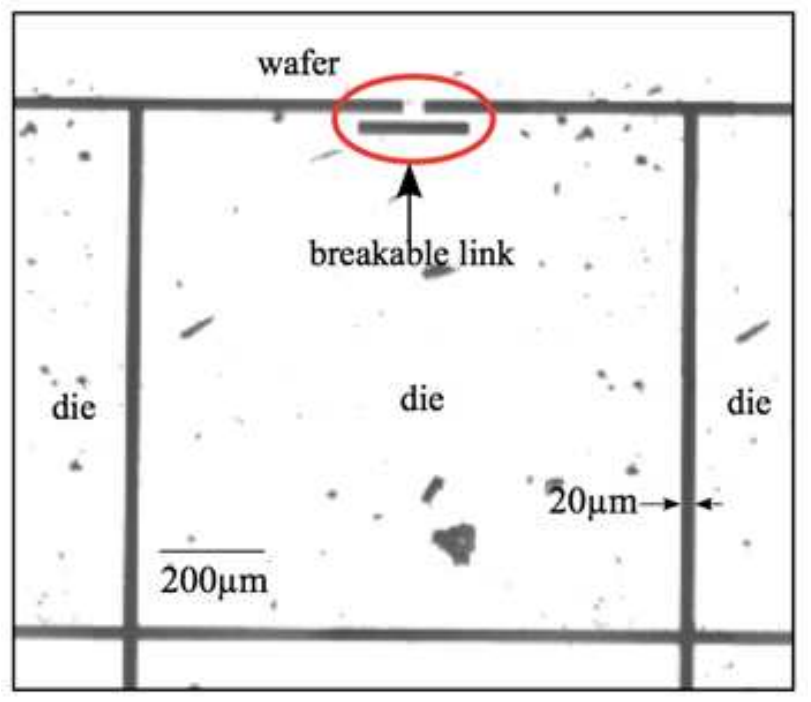

Figure. 1. Optical microscope image of an ultra-thin die with $10 \mu \mathrm{m}$ thickness and $1 \times 1 \mathrm{~mm}^{2}$ area, supported on the wafer by the breakable link.
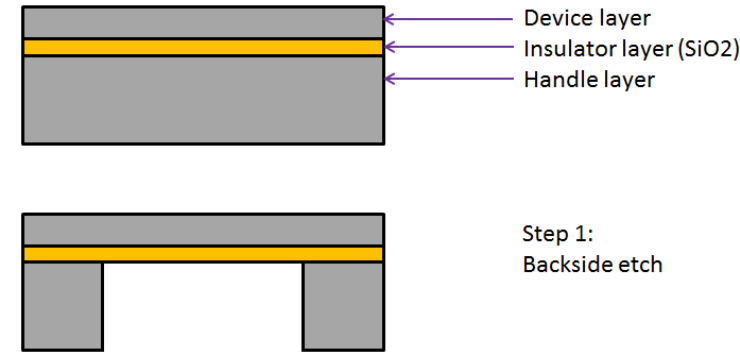

Step 1:

Backside etch

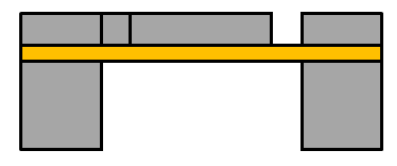

Step 2:

Device layer etch

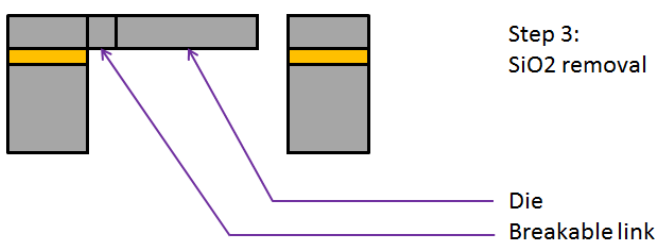

Figure. 2. Fabrication steps for ultra-thin dies with a breakable link on SOI wafer.

\section{Normal force applied in} the center of the die $(\mathrm{mN})$

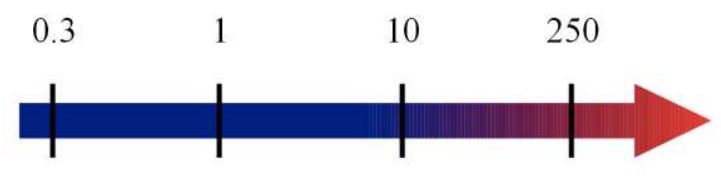

Fabrication

Vacuum

$\begin{array}{ccc}\text { Breakable } & \text { gripping } \\ \text { link rupture } & \text { Die rupture }\end{array}$

Figure. 3. Mechanical stress constraints for dies with breakable links.

\section{A. Mechanical characterization of breakable links}

Several types of breakable link structures were designed and fabricated for a fixed die area of $1 \mathrm{~mm} 2$ while both $10 \mu \mathrm{m}$ and $5 \mu \mathrm{m}$ thicknesses were tested. Strength of the link designs were both simulated (Comsol Multiphysics 3.5) and measured. The fabrication process and especially wet etching induced significant forces which could break the link. The process (Fig. 2) was tested on a test wafer with a large variety of links. We observed that the links whose simulated breaking force is lower than $0.3 \mathrm{mN}$ could be broken during the fabrication process. Consequently, we assume that the fabrication process is able to apply a maximal force of 0.3 $\mathrm{mN}$. In order to measure the breaking force, a normal force was applied with a force sensor (Femto-Tools FT-S 1000) in the center area of the die (Fig. 4). For the breakable link shown in Fig. 1, simulation predicted a rupture force of $1 \mathrm{mN}$, which was in good agreement with the average measured rupture force of $1.5 \mathrm{mN}$. Fig. 5 shows a broken link after pick-up from the wafer, with the rupture safely confined outside of the die. 


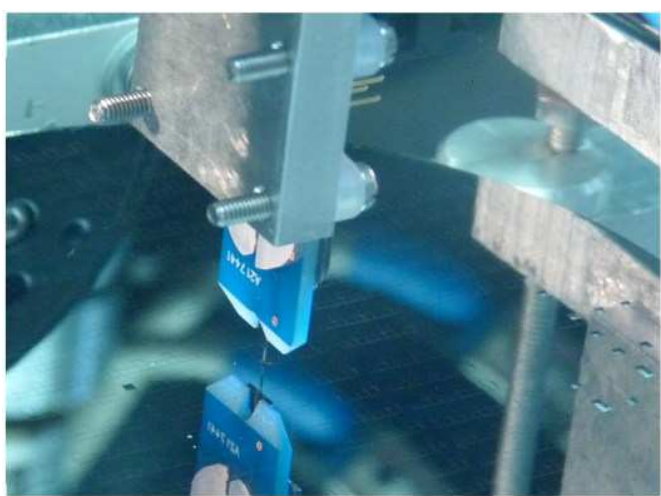

Figure. 4. Measurement of breakable link strength by applying normal force with a force sensor in the die center.

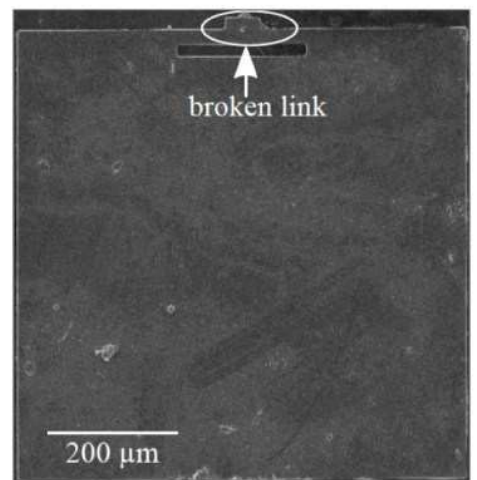

Figure. 5. Detached die with a broken link after pick-up from the wafer.

As shown in Fig. 3, the gripper should be able to handle dies with a force lower than the rupture force of the dies, which has been simulated by finite element method to reach $250 \mathrm{mN}$ (Comsol Multiphysics 3.5). To ensure such a low handling force, a vacuum gripper with controllable suction force was developed.

\section{B. Handling of ultra-thin dies}

The working principle of the developed vacuum gripper is presented in Fig. 6. Air is pumped from the gripper head when an electro-valve between the gripper and the pump is open. There is also a leak hole in the gripper head, which allows suction force on the die to be controlled. Gripper heads with different leak hole sizes were designed. The suction pressure can be as low as $10 \mathrm{kPa}$, ensuring safe handling of the dies.

Pick-and-place of the dies is performed using a robotic micro-assembly station shown in Fig. 7. The system consists of an $\mathrm{x}$-axis positioning stage (Physik Instrumente M404.8PD, $0.25 \mu \mathrm{m}$ accuracy), a y-axis positioning stage (Physik Instrumente M-122.2DD, $0.1 \mu \mathrm{m}$ accuracy), a vertical positioning stage where the vacuum gripper is mounted (Physik Instrumente M-122.2DD, $0.1 \mu \mathrm{m}$ accuracy), a water dispenser (Gesim PicPIP) and microscopes for top, side and angled views. The top view microscope (Edmund Optics VZM1000i) is attached to a high speed, progressive scan CCD camera (Imperx IGV-B0620M), and the side view microscope (Edmund Optics VZM1000i) is also attached to a high-speed CCD camera (Imperx IGV-B1620C). At the other

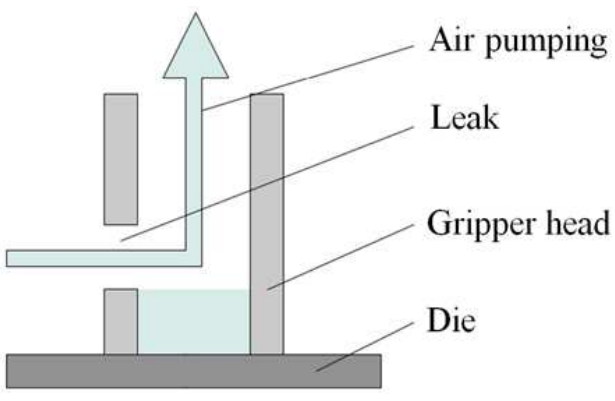

Figure. 6. Vacuum gripper working principle. Suction force can be altered by changing the size of the leak hole.

end of the station, a second side view microscope (Edmund Optics VZM600i) is attached to a high-speed CCD video camera (Imperxl IGV-B1620M). There is also a microscope (Edmund Optics VZM600i) providing angled view, attached to a progressive scan camera (Sony XC-8500). Droplet dispensing is done at one end of the station (Fig. 7, back) assisted by the top view, and pick-and-place at the other end (Fig. 7, front).

\section{EXPERIMENTAL}

In order to experimentally validate feasibility of the hybrid handling approach (sketched in Fig. 8) for stacking of ultra-thin dies, tests with different process parameters were performed. The basic procedure is as follows: an ultra-thin die is picked up by the gripper (Fig. 9), water droplet(s) are dispensed on the die at the top of the stack, the picked die is released above the stack and capillary force aligns the die with the die on top of the stack (Fig. 10). The desired accurate self-alignment is defined as no visible alignment error with respect to the other dies of the die stack in optical microscope images (sub-micron accuracy). The optically determined accuracy was also verified in SEM for the large die stacks (12 dies).

Two different aspects of this procedure were studied: 1) the parameters related to the droplets on the top of a die stack, including the number of droplets, the volume of the droplets, and the distribution of the droplets; 2) the effect of biases in the die releasing position and orientation.

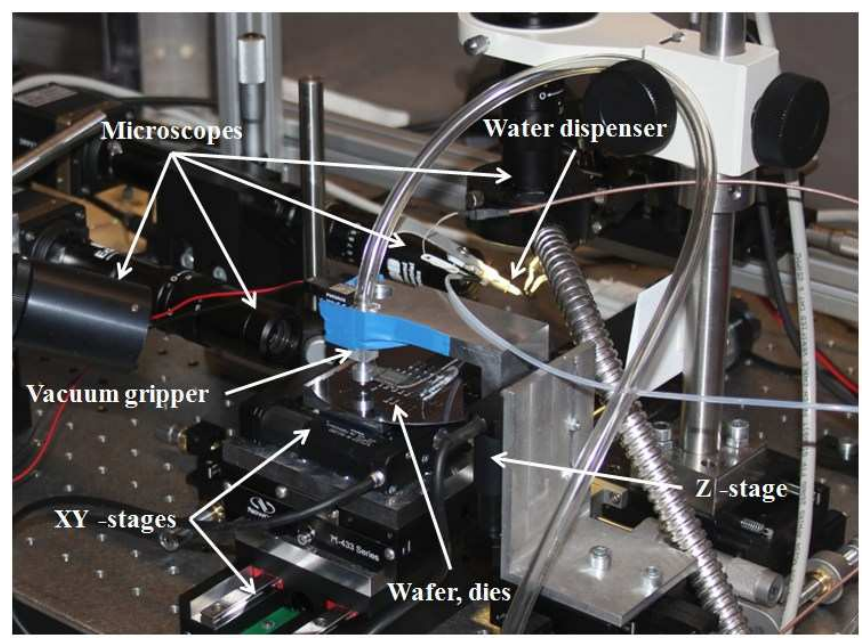

Figure. 7. Robotic micro-assembly system used for handling of ultra-thin dies. 


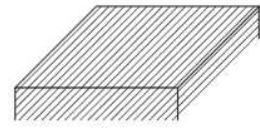

a)

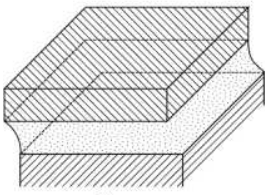

d)

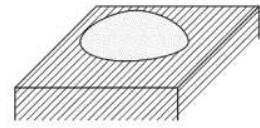

b)

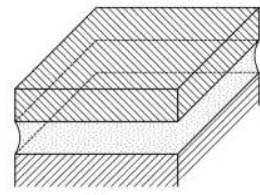

e)

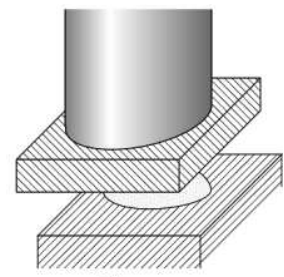

c)

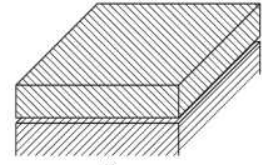

f)

Figure. 8. Concept of hybrid microassembly: a) receptor site, e.g. a die, b) liquid, e.g. water, is dispensed on the receptor site, c) a pick-and-place tool such as a vacuum gripper brings a picked die on top of the receptor site, d) the picked die is released on top of the receptor site and a water meniscus is formed between the parts, e) capillary forces of the water droplet minimize potential energy of the droplet, self-aligning the top die on the receptor site, and f) water evaporates and the parts are left in contact.

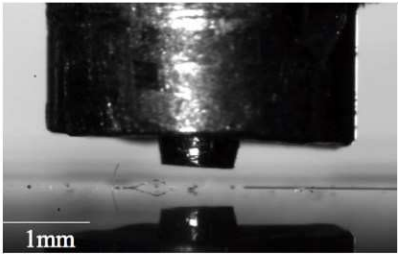

(a)

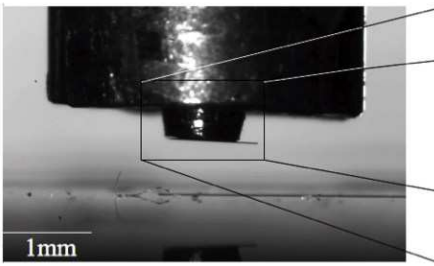

(c)

Figure. 9. Die detachment from the wafer: (a) approach, (b) breaking the link by pushing, and (c) grasping the die by suction.

\section{A. Water droplet influences}

The influence of the number of water droplets, the volume of droplets and droplet distribution has been studied against the final position of the released dies, i.e. accuracy of the alignment, and the duration of the self-alignment process. To avoid influences from other parameters, the dies were released directly on the top of the stack without intentional bias. For the droplet influence tests, three cases were studied.

In the first case, one small droplet was dispensed in the center of the die, covering about $25 \%$ of its surface (Fig. 11(a)), with an estimated volume of $7 \mathrm{nl}$. After the die was placed on top of the droplet, the droplet spread due to wetting. However, such wetting process stops at the cavity of the breakable link structure (Fig. 11(a)) due to the edge pinning the water [12]. Consequently, such wetting leads to an asymmetric total wetted surface relative to the geometrical center of the die at the top of the stack, and clear misalignment can be observed consistently (Fig. 11(b)).

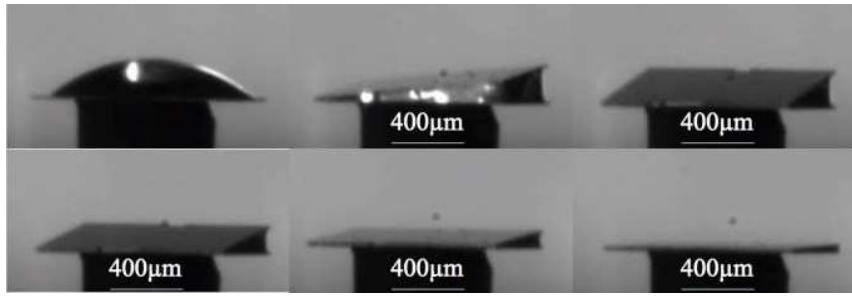

Figure. 10. Self-alignment of an ultra-thin die on top of another die.

Evaporation of the water took about 90 seconds due to the large droplet volume.

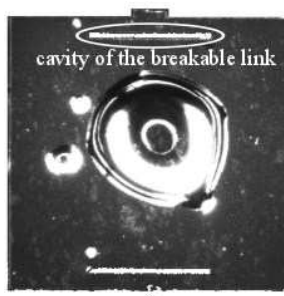

a)

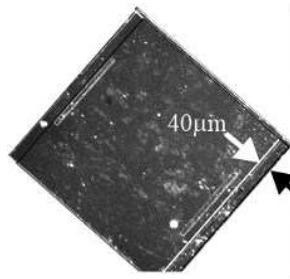

b)

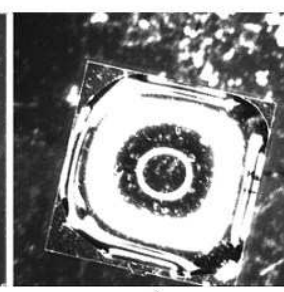

c)

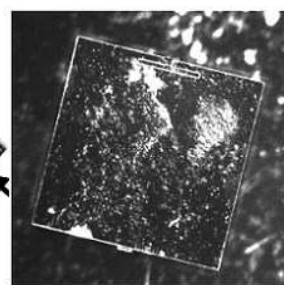

d)

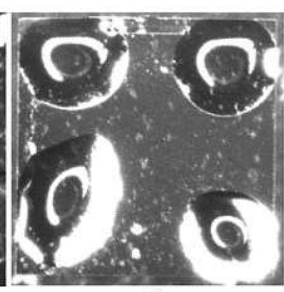

e)

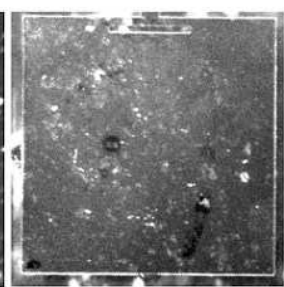

f)
Figure. 11. Effect of the droplet volume and number on self-alignment.

In the second case, a larger droplet (estimated volume $54 \mathrm{nl}$ ) was dispensed in the center, which covers more than $80 \%$ of the die surface, including the breakable link structures. The droplet is confined only by the edges of the stack top die (Fig. 11(c)) and the water completely bridges the cavity of the breakable link. Therefore, self-alignment was governed by the edges of the die under the droplet and was accurate (Fig. 11(d)). However, evaporation of the water droplet takes a longer time with increased volume (e.g. 90 seconds for the droplet in Fig. 10). Therefore, using a large droplet is suboptimal regarding process throughput.

In the third case, we dispensed four small droplets near the corners of the die, each covering about $10 \%$ of the surface area and having a volume of approximately $3 \mathrm{nl}$, see Fig. 11(e). Due to the position of the droplets, the cavity of the breakable link does not hinder wetting when a die is placed on top of the droplets. Therefore the droplet will wet the whole die area during self-alignment and the alignment is accurate as expected (Fig. 11(f)). Furthermore, water evaporation time with the four-droplet configuration is significantly reduced to approximately one second, giving a clear advantage over the single-droplet case.

\section{B. Release position and angular biases}

The second part of the experiments studies the effect of biases in the release position on the alignment accuracy. The independently studied parameters are: $\mathrm{x}$ - and $\mathrm{y}$-bias, $\mathrm{z}$-bias (release height) and angular bias $\theta$ (die rotation). These parameters are illustrated in Fig. 12. 


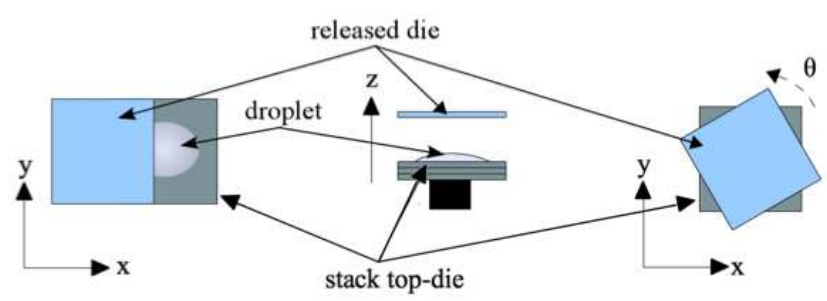

Figure. 12. Different biases applied to the die release position and orientation: $\mathrm{x}-, \mathrm{y}-, \mathrm{z}-$ (height) and $\theta$ (angular) bias.

Based on earlier extensive results on hybrid microassembly [5], droplet based self-aligment of microchips is almost a binary process - a parameter set for successful self-alignment will very reliably lead to success every time, and vice versa. Therefore, a well-planned single test gives a good indication of whether self-alignment will work or not. In the experiments, the four-droplet approach was chosen as dispensing strategy due to its advantage in alignment speed, as discussed above.

1) Effect of $x$ - and $y$ - bias: As the die outline is symmetric with respect to the $\mathrm{x}$ - and $\mathrm{y}$-axes, only $\mathrm{x}$-direction was considered in these tests. Release height (z) was fixed at $50 \mu \mathrm{m}$ and the angular bias $\theta$ was kept as close to zero as possible. The tests showed that for the $1 \mathrm{~mm} \times 1 \mathrm{~mm}$ dies selfalignment was accurate up to a $900 \mu \mathrm{m}$ bias in the $\mathrm{x}-$ direction (Fig. 13, top row). Beyond that limit, top of die to be placed was wetted as well and self-alignment failed (Fig. 13 , bottom row). The biases tested were $620 \mu \mathrm{m}, 720 \mu \mathrm{m}$, $770 \mu \mathrm{m}, 900 \mu \mathrm{m}$ and $950 \mu \mathrm{m}$.

2) Effect of z-bias: Influence of release height on selfalignment was tested by varying it along the $\mathrm{z}$ - direction with no other biases present. Tests with the release height ranging from $0.4 \mathrm{~mm}$ to $1.25 \mathrm{~mm}$ showed that alignment was successful up to a distance of $1 \mathrm{~mm}$ from the stack top die surface (Fig. 14, top row). With larger release heights the die may fall too far from the receiving die for self-alignment to work (Fig. 14, bottom row). The biases tested were $0.4 \mathrm{~mm}, 0.7 \mathrm{~mm}, 0.9 \mathrm{~mm}, 1 \mathrm{~mm}$ and $1.25 \mathrm{~mm}$.

3) Effect of angular bias: Angular bias $\theta$ was applied with values of 0,30 and 40 degrees. At 45 degrees there is a local maximum of potential energy due to symmetry.

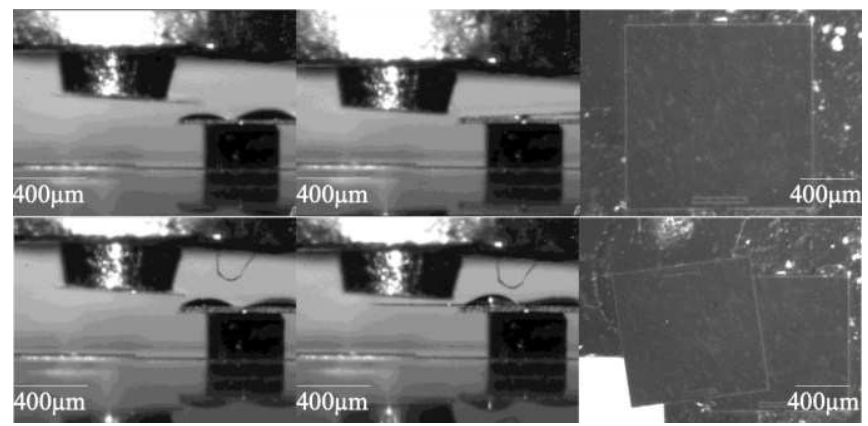

Figure. 13. Effect of $x$-bias on self-alignment of $1 \times 1 \mathrm{~mm}^{2}$ ultra-thin die: (top row) accurate self-alignment with $900 \mu \mathrm{m} x$-bias, (bottom row): failed self-alignment with $950 \mu \mathrm{m} x$-bias.

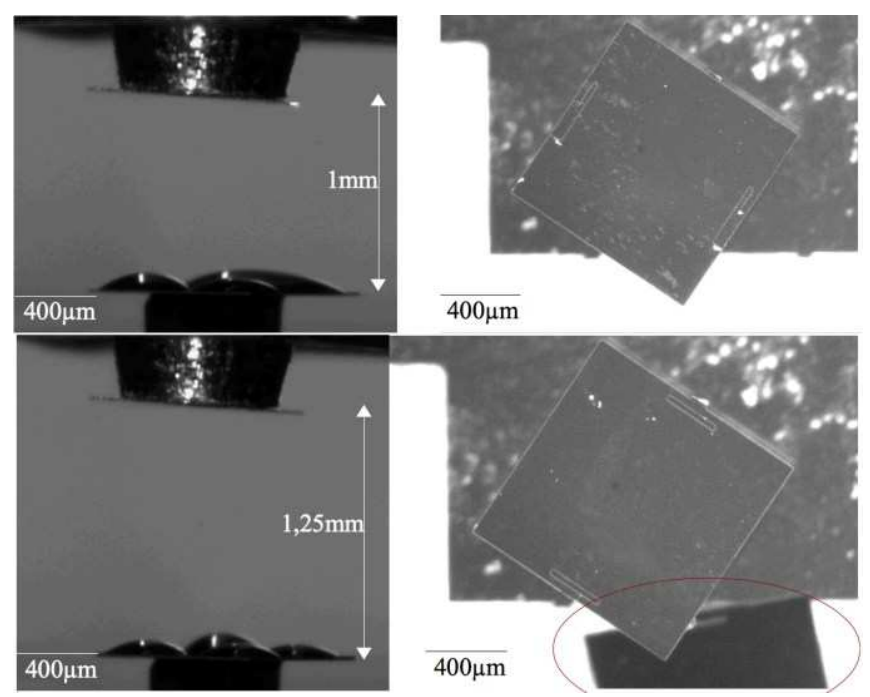

Figure. 14. Effect of z-bias (release height) on self-alignment of ultra-thin dies: (top row) self-alignment succeeds up to $1 \mathrm{~mm}$ release height, (bottom row) self-alignment fails at $1.25 \mathrm{~mm}$ release height due to the die falling past the stack top die.

Release height was fixed at $50 \mu \mathrm{m}$ and there was no other bias. Alignment was accurate in all the tests, showing that rotation of the die alone does not impede self-alignment.

Further tests were performed by combining lateral biases with angular bias, within the limits of each bias obtained earlier. Alignment succeeded accurately in all the tests. This confirms that capillary self-alignment is a feasible method for accurate stacking of $10 \mu \mathrm{m}$ thick dies. Relatively large inaccuracies can be allowed in the initial release position without affecting the final alignment accuracy.

\section{Micro-Assembly of Ultra-Thin Dies}

The experiments described so far have been done one by one with teleoperation control of the positioning systems, dispenser and vacuum gripper. In this section, automatic stacking of 3 dies and semi-automatic stacking of up to 12 ultra-thin dies are reported.

Automation of the ultra-thin die stacking was done to demonstrate the relevance to real-world applications. The procedure is rather straightforward. As the dies are located on the wafer with regular spacing, they can be easily picked and stacked sequentially by incrementing the picking position coordinates after each release. The release position can be maintained fixed or incremented as needed for different stack heights. A stack of 3 ultra-thin dies was assembled using this method. Stacking more dies automatically will be straightforward. However, it has not been done because the dispenser is not installed on a motorized stage in the current setup. In the tests, only one large droplet was dispensed in each cycle, and the result shows that the droplet from previous cycle need not be evaporated for the self-alignment of the next die to work. The automatic stacking of 3 dies took about 40 seconds, due to long-range transportation with low-speed positioning stages between the dispensing and vacuum gripping units of the hybrid assembly station (Fig. 7). The process can be improved by using a state-of-the-art pick-and-place machine, and the resulting throughput can be expected to be better than assembly without self-alignment, because no 
time-consuming machine vision based fine positioning is needed.

The stacking of larger number of dies has also been done using teleoperation. Firstly, stacks of two dies were grasped and self-aligned on top of each other to form stacks of 4 dies. The adhesion force between two stacked dies due to the capillary force that also aligned them, allows the handling of those 2-die stacks as one unit. The stacks of 4 dies were then assembled similarly to form a stack of 8 dies (Fig. 15), and finally 12 dies. An SEM image of the stack of 12 dies shows high accuracy of the assembly (Fig. 16).

\section{CONCLUSION}

We have proposed a handling and assembly strategy for ultra-thin $10 \mu \mathrm{m}$ dies, which is clearly beyond the thickness found in state-of-the-art microassembly. The dies are built with breakable links, allowing safe pick-up without damage to the dies directly from the wafer instead of an intermediate carrier tape. A vacuum gripper with controllable suction force was developed for the pick-and-place task. Accurate stacking of the dies is based on capillary self-alignment of a water droplet. Experimental results show sub-micron alignment accuracy, proving the feasibility of the hybrid assembly strategy for the assembly of ultra-thin dies. However, we note that the achievable alignment accuracy is dependent on the die fabrication accuracy, and contamination from environment may have an impact on contact line of the self-alignment liquid [13], possibly affecting the alignment accuracy. Nevertheless, the approach

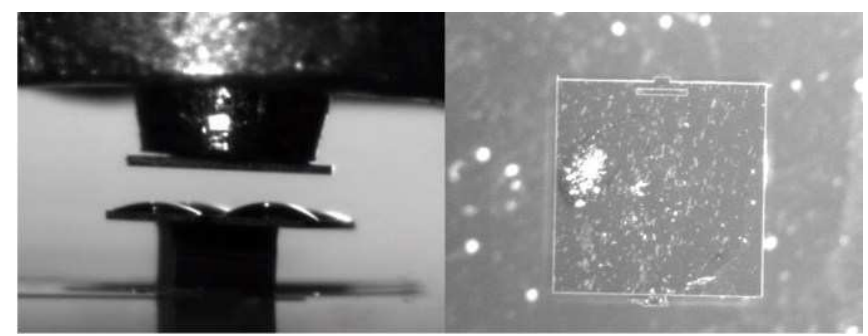

Figure. 15. Self-alignment of 2 stacks of 4 ultra-thin dies each to a stack of 8 dies.

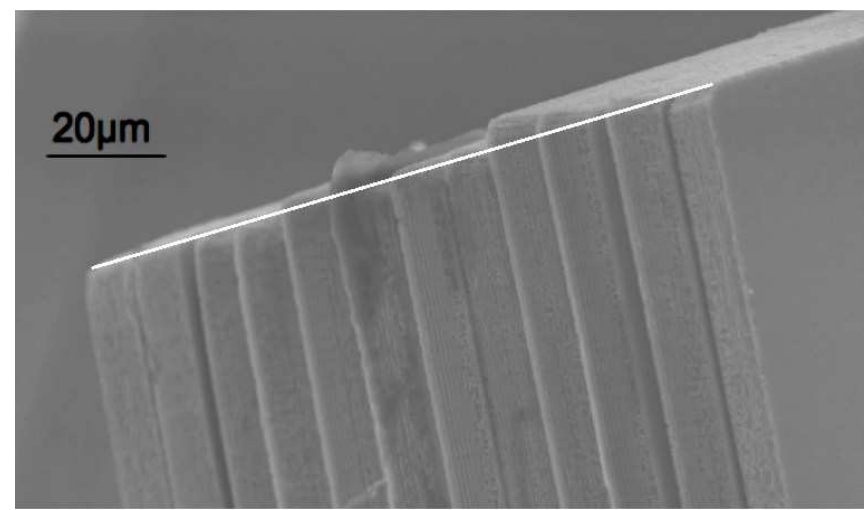

Figure. 16. An SEM image of stack of 12 ultra-thin dies. The white line indicates alignment of the die corners. has been shown to be very robust to placement inaccuracies of up to $90 \%$ of the die size. A fully automatic assembly process of several dies has also been demonstrated.

The dies used in this work were plain silicon without any functionality. Future research on the topic will include dies with micro-bumps, which is the case in real-world 3D integration of die stacks. It is expected that capillary selfalignment will also work for functional dies with bumps as long as the droplet is confined by the die edges and not by some other features on the die. Chemical functionalization of certain areas may be a solution in the latter case.

\section{ACKNOWLEDGMENT}

The authors would like to thank all the contributors in this collaborative work under the European project FAB2ASM (contract FoF-NMP-2010-260079): Efficient and Precise 3D Integration of Heterogeneous Microsystems from Fabrication to Assembly, (http://fab2asm.eu/).

\section{REFERENCES}

[1] (2011) 'International Technology Roadmap for Semiconductors' [Online]. Accessed on $14^{\text {th }}$ September, 2012. Available: http://www.itrs.net/Links/2011ITRS/Home2011.htm

[2] B. Wu, A. Kumar and S. Ramaswami. 3D IC Stacking Technology. McGraw-Hill Book Publication, 2011.

[3] V. Sariola, Q. Zhou, and H. Koivo, "Three dimensional hybrid microassembly combining robotic microhandling and self-assembly," in 2009 IEEE Internation Conference on Robotics and Automation, ICRA'09, pp. 2605-2610, Kobe, Japan, 2009.

[4] B. Chang, V. Sariola, M. Jääskeläinen, and Q. Zhou, "Self-alignment in the stacking of microchips with mist-induced water droplets," Journal of Micromechanics and Microengineering, vol. 21, no. 1, p. 015016, 2011

[5] V. Sariola, M. Jääskeläinen, and Q. Zhou, "Hybrid microassembly combining robotics and water droplet self-alignment," in IEEE Transactions on Robotics, vol. 26, no. 6, pp. 965-977, 2010.

[6] Q. Zhou and B. Chang, "Microhandling using robotic manipulation and capillary self-alignment," in 2006 IEEE International Conference on Intelligent Robots and Systems, IROS'06, Beijing, China, 2006.

[7] B. Chang, M. Jääskeläinen, and Q. Zhou, "Hybrid micro assembly of microchips on segmented patterns," in 2010 IEEE International Conference on Automation Science and Engineering, pp. 15- 20, 2010.

[8] J. N. Burghartz, W. Appel, H.D. Rempp, and M. Zimmermann, "A new fabrication and assembly process for ultrathin chips," in IEEE Transactions on Electron Devices, vol. 56, no. 2, pp. 321-327, 2009.

[9] K. Gurnett and T. Adams, "Ultra-thin semiconductor wafer applications and processes," Advanced Semiconductor Magazine, vol. 19, no. 4, pp. 38-40, 2006.

[10] W. Kröninger and F. Mariani, "Thinning and singulation of silicon: Root causes of the damage in thin chips," in IEEE Electronic Components and Technology Conference, ECTC'06, San Diego, USA, 2006.

[11] D. Hériban, J. Agnus, V. Pétrini, and M. Gauthier, "Mechanical detethering technique for silicon MEMS etched with dry process," Journal of Micromechanics and Microengineering, vol. 19, p. 055011, 2009.

[12] J. F. Oliver, C. Huh and S. G. Mason, "Resistance to spreading of liquids by sharp edges," Journal of Colloid Interface Science, vol. 59, pp. 568-581, 1977.

[13] A. S. Sangani, C. Lu, K. Su, and J. A. Schwarz, "Capillary force on particles near a drop edge resting on a substrate and a criterion for contact line pinning" Physical Review E, vol. 80, 011603, 2009. 